\title{
Aferição de usabilidade de interfaces assistivas sob o prisma da com- putação afetiva
}

\author{
Measuring the Usability of Assistive Interfaces from the Affective Computing \\ Prism
}

\author{
Rovilson Mezencio ${ }^{*}$, Rodrigo Pinto Lemos ${ }^{1}$,Ana Carolina do C. Andrade ${ }^{2}$
}

\section{RESUMO}

Testes psicométricos podem classificar pessoas dentro de grupos com habilidades, competências, modo de agir e pensar semelhantes. Interfaces de software assistivas deveriam ser personalizadas ao máximo, a fim de reduzir o impacto do uso da tecnologia por pessoas com deficiências ou seus cuidadores. Seria relevante saber se personalidades diferentes reagem emocionalmente ou mesmo cognitivamente da mesma forma ao usar uma ferramenta assistiva. Em uma pesquisa realizada com 98 participantes, autorizada pelo Comitê de Ética da UFG, é isso que este trabalho propõe a descobrir.

Palavras Chave: Computação Afetiva; Software Assistivo; Testes Psicométricos; Interface Homem Máquina; Comunicação Alternativa e Aumentativa.

\begin{abstract}
Psychometric tests can classify people into groups with similar abilities, competences, ways of acting and thinking. Assistive software interfaces should be as much as possible customized in order to reduce the impact of technology use by people with disabilities or their caregivers. It would be relevant to know whether different personalities react emotionally or even cognitively in the same way when using an assistive tool. In a survey made with 98 participants, authorized by the UFG Ethics Committee, this is what this work try to discover.
\end{abstract}

Keywords: Affective Computing; Assistive Software; Psychometric Tests; Man-machine interface; Alternative and Augmentative Communication.

${ }^{1}$ Escola de Computação e Engenharia Elétrica da UFG, Universidade do Estado de Goiás.

* E-mail: rovilsonm@gmail.com

${ }^{2}$ Programa Interinstitucional de Pós-Graduação em Estatística UFSCar-USP (PIPGEs) 


\section{INTRODUÇÃO}

Segundo a OMS, com dados de 2011, um bilhão de pessoas vivem com alguma deficiência, isso significa uma em cada sete pessoas no mundo. A falta de estatísticas sobre pessoas com deficiência é um obstáculo para planejar e implementar políticas de desenvolvimento que melhoram as vidas das pessoas com deficiência (NU,2021).

A ONU alerta ainda que $80 \%$ das pessoas que vivem com alguma deficiência residem nos países em desenvolvimento. No total, 150 milhões de crianças (com menos de 18 anos de idade) tem alguma deficiência, segundo o UNICEF(NU,2021).

Dados do Censo 2010, último censo no Brasil, revelaram que cerca de $24 \%$ da população brasileira o que equivale à 45,6 milhões de pessoas, na época, possuíam algum tipo de deficiência [INSTITUTO BRASILEIRO DE GEOGRAFIA E ESTATÍSTICA, 2021].

\section{CONTEXTUALIZAÇÃO}

Essas pessoas com restrições motoras e de fala simultâneas têm a comunicação verbal e a linguagem corporal prejudicadas. Nos casos mais extremos o paciente é privado de todos os seus movimentos e da capacidade de fala. Essa situação é caracterizada como a Síndrome do Encarceramento (SE). Nestes e em outros casos onde a comunicação fica prejudicada a Comunicação Aumentativa e Alternativa (CAA) através de software de tecnologia assistiva podem ajudar (PARREIRA, DOS SANTOS, et al., 2015).

A Interface Homem Máquina- IHC é uma área da ciência que estuda o desenvolvimento de interfaces mais amigáveis ao ser humano. Mais amigáveis está conectado diretamente à qualidade de uso que é relacionado à capacidade e a facilidade de os usuários atingirem suas metas com eficiência e satisfação. É preciso que o usuário tenha vias alternativas para as tarefas o fato de o usuário sentir-se à vontade para usar trilhas dentro do sistema está coligado à vontade ou não de usar o software com certa frequência. Em suma vontade de usar com frequência é igual a qualidade de interface que remete a ser amigável (ABRAS, MALONEY-KRICHMAR, et al., 2004).

Um software assistivo precisa ser mais amigável e personalizado possível quanto às necessidades de cada um bem como à personalidade de cada usuário afim de 
tornar muito eficiente e produtivo a interação homem máquina (PEREIRA, 2013).

A CAA é uma área de estudo altamente multidisciplinar. Psicólogos, Fisioterapeutas, Terapeutas Ocupacionais, Psicopedagogos, Médicos, Fonoaudiólogos e desenvolvedores de software assistivo dentre outros colaboram entre si (CESA e MOTA, 2015).

Estudos multidisciplinares: psicólogos, neurologistas, antropólogos e cientistas computacionais (DAMASIO, 1995) (TRAPPL e PETTA, 2001) ( (PICARD, 2000) têm demonstrado que aspectos psicológicos humanos, tais como emoção e personalidade devem ser levados em conta em projetos de interfaces.

Adequar a interface do software assistivo ao perfil psicoafetivo do usuário, particularmente no caso de necessidades especiais de comunicação é algo novo e apesar de sua relevância clara, ainda poucos trabalhos relacionados vêm sendo encontrados (NUNES, 2012).

Dentro desse contexto e olhando para a uma área da computação conhecida como computação afetiva (BERCHT, 2006), este trabalho vai estudar a correlação entre a percepção do usuário sobre a usabilidade da interface de softwares assistivos e os perfis psicológicos desses usuários, aferidos por meio de método psicométrico baseado no Big Five o ICP - Inventário de Características da Personalidade, criado e validado no Brasil (GOMES, 2012).

Uma vez classificado em uma das oito personalidades do ICP, será usado o GEW - The Geneva Emotion Wheel (SACHARIN, SCHLEGEL e SCHERER, 2012) para medir como a interface assistiva afetou a emoção de cada perfil do ICP. E por fim, será usado o SUS - System Usability Scale (LEWIS, 2018) afim de medir como cada personalidade foi afetava cognitivamente.

\section{HIPOTESES}

A pesquisa procura descobrir se além dos testes clássicos da literatura de engenharia de Software, no que se refere à usabilidade de interfaces assistivas, a personalidade e as emoções deveriam ser consideradas em um processo de testes e bem como, se as oito personalidades do ICP-Inventário de Características da Personalidade (GOMES, 2012) 
tem reações cognitivas e emocionais semelhantes quando usam tecnologia assistiva. Para afirmar estas premissas, foram escritas três hipóteses:

H1. Usuários com o mesmo tipo de personalidade apresentam a mesma percepção emocional sobre a usabilidade de interfaces assistiva.

H2. Usuários com o mesmo tipo de personalidade apresentam a mesma percepção cognitiva sobre a usabilidade de interfaces assistiva.

H3. A percepção emocional e cognitiva, no uso de uma ferramenta assistiva, tem correlação.

\section{PSICOMETRIA E O ESTUDO DAS PERSONALIDADES}

Em nosso dia a dia é possível encontrar vários tipos de medidas: massa, distância, peso, altura, densidade, escalas espaciais e partículas microscópicas dentre outras a psicometria é uma área da psicologia que usa medidas, baseada em testes para ajudar pessoas a se conhecerem e serem ajudadas (ANDRIOLA, 2009).

Seja no que se refere à aptidão, intelecto, personalidade ou emoções a psicometria através de processos matemáticos/estatísticos consegue constatar diferenças entre indivíduos. Sir Francis Galton (1822-1911), é reconhecido como o primeiro cientista a estudar medição de diferenças individuais (GILLHAM, 2001)

Em seguida veio Alfred Binet (1857-1911), psicólogo francês que criou a escala métrica de Binet-Simon, Simon era seu auxiliar. Essa escala é capaz de aferir dois conceitos fundamentais: idade cronológica e idade mental, sendo que nível de desenvolvimento mental que pode ser inferior, igual ou superior à idade cronológica (SIEGLER, 1992).Como base nas pesquisa de Binet, William Stern, psicólogo e filósofo criou o teste de Quociente Inteligente-Q.I (LAMIELL, 1996).

Sensibilidade, validade e fidelidade são os três fatores que todo teste psicométrico precisa obedecer. Sensibilidade é o poder de classificação de um teste, se um teste classifica apenas em duas polaridades, por exemplo: Bom ou Mal, Fraco ou Forte. Este teste tem o critério de sensibilidade muito fraco (DESOUSA, MORENO, et al., 2013).

Validade é a capacidade que um teste tem de medir aquilo ao que se destina. 
Por exemplo se um teste se propõe a medir a inteligência de um grupo de alunos. Medir a força física, ou sua altura não cabem dentro do contexto desse teste. Fidelidade resumese na ideia de que se o mesmo indivíduo fizer o mesmo teste duas vezes o resultado deverá ser igual (DESOUSA, MORENO, et al., 2013).

Dentre os mais variados testes psicométricos dois deles vêm sendo usados em trabalhos multidisciplinares onde perfis e usabilidade são estudados em conjunto: MBTI® Myers-Briggs Type Indicator (BRIGGS, 1976)e o Big Five (NUNES, HUTZ e NUNES, 2010).

O MBTI, que mapeia dezesseis personalidades, também deve ser lembrado por se tratar de um instrumento de identificação de características pessoais que possibilita identificar as características, pontos fortes e aspectos de desenvolvimento.

O Big Five, ou Modelo dos Cinco Grandes Fatores, foi desenvolvido através de estudos da Teoria dos Traços de Personalidade, para mapeamento das dimensões humanas básicas. McDougall, na década de 30, foi um dos pioneiros na pesquisa do modelo, a ideia inicial era que a análise da linguagem de parte de uma população ajudaria a entender e classificar a sua personalidade (FURNHAM, CHAMORRO-PREMUZIC e MCDOUGALL, 2003).

\section{TESTE PSICOMETRICO ESCOLHIDO}

Com base nas ideias em torno do Big Five, surgiram vários inventários. Inventário aqui quer dizer: um conjunto de perguntas, frases, afirmações qual foi testado, validado e publicado em alguma língua (LIMA, 1997).

Em geral os testes psicométricos são baseados em autorrelatos. As pessoas leem uma frase, afirmação ou até um adjetivo e se auto classificam se concordam ou não com aquele texto, normalmente dentro de uma escala de Likert (CUNHA e OTHERS, 2007).

Dentro desse contexto é preciso que o teste original criado em outra língua seja traduzido para o português e validado com pesquisa publicada, dessa forma baseados no Big Five foram validados no Brasil alguns testes dentre eles os mais conhecidos: 
NEO PI-R - Neuroticism, Extraversion, Openness to Experiences Personality Inventory Revised, Autora Prof. Dr. Carmem Flores -Direitos autorias da Editora Vetor (FLORES-MENDOZA, 2007), número de questões 240 (duzentas e quarenta);

BFP - Bateria Fatorial de Personalidade, Autor Prof. Dr. Nunes (NUNES, HUTZ e NUNES, 2010), Direitos autorias da Editora Pearson, número de questões 126 (cento e vinte e seis);

IGFP-5 Inventário dos Cinco Grandes Fatores de Personalidade (ANDRADE, 2008), uso deve ser autorizado pelo autor, número de questões 41 (quarenta e uma);

ICFP-R- Inventário Reduzido dos Cinco Grandes Fatores de Personalidade (TRÓCCOLI, VASCONCELOS, et al., 2001), uso deve ser autorizado pelo autor, número de questões 117 (cento e dezessete); e o

ICP-Inventário de Características da Personalidade (GOMES, 2012), uso deve ser autorizado pelo autor, número de questões 27 (vinte e sete).

Nossa escolha foi o ICP, e ela foi feita com base no seguinte critério: Mapear o maior número de polaridades com menor número de questões usando um teste de eficiência comprovada em português, gratuito cujo o autor autorizasse o uso.

O ICP mapeia oito polaridades, das dez, do Big Five, usando apenas vinte e sete questões, foi validado em pelo menos dois trabalhos diferente e nos foi autorizado pelo Prof. Dr. Cristiano Mauro Assis Gomes, da Universidade Federal de Minas Gerais, do Programa de Pós Graduação em Neurociências (GOMES, 2012).

Marsh (MARSH, MORIN, et al., 2014) afirma que nenhum questionário de autorrelato dos cinco grandes fatores apresenta um grau aceitável de ajuste na análise fatorial confirmatória (CFA). Com base nessa afirmação, além do trabalho que validou o ICP no Brasil ( (GOMES, 2012) foi realizado um novo estudo com os dados do estudo original criou.

Usando abordagens análises fatoriais exploratórias (AFE) afim de concluir se o ICP apresentava grau aceitável da CFA (GOMES e GJIKURIA, 2017). A conclusão foi que sim o ICP tem grau de CFA aceitável. 
Sendo o mais moderno com confirmação de aceitação em português, sem necessidade de licença para uso e como menor número de perguntas (vinte e sete) o ICP foi o escolhido neste trabalho, para classificar as personalidades dos participantes.

\section{AFERIÇÃO DO IMPACTO DA USABILIDADE NA COGNIÇÃO}

A ideia de testar usabilidade olhando para o lado cognitivo já existe desde 1996 (SMITH e MAYES, 1996; NIELSEN, 1990). Dentre os testes mais antigos e eficientes podemos mencionar o SUMI (Software Usability Measurement Inventory) (KIRAKOWSKI e CORBETT, 1993) e o QUIS (Questionnaire for User Interface Satisfaction) (HARPER e NORMAN, 1993).

O QUIS apresenta quatro fatores: aprendizagem, terminologia e fluxo da informação, saídas do sistema e características do sistema. É composto por dezessete perguntas e usa uma escala de diferencial baseado em palavras, semântico.

O SUMI foi desenvolvido pelo Human Factors Research Group, em Cork, e é constituído por cinco fatores: gosto, eficiência, aprendizagem, ajuda e controle. Com cinquenta itens o SUMI e utiliza uma escala de Likert (LIKERT, 1932) de três pontos.

No caso deste trabalho a avaliação será de um site (DESOUSA, MORENO, et al., 2013) o SCALA. Dessa forma métodos de avalição com grande quantidade de perguntas não vão contribuir. Um estudo comparativo aprofundado sobre testes de usabilidade (ASSILA, EZZEDINE e OTHERS, 2016) recomenda que neste caso, uso de site, o melhor e mais simples teste seria o SUS - System Usability Scale (LEWIS, 2018).

No trabalho de (LEWIS, 2018) a eficiência do SUS para avaliação de sites é demonstrada. Confiável e fácil de usar o SUS possui apenas dez perguntas simples, em uma escala Likert de zero a cinco.

Além disso minimizar o número de perguntas, sem perder a qualidade é claro, é um dos objetivos deste trabalho. Assim sendo o SUS é duplamente adequado à este trabalho.

\section{AFERIÇÃO DO IMPACTO DA USABILIDADE NAS EMOÇÕES}


A computação afetiva (MORAIS, DA SILVA, et al., 2017) surge com o objetivo de criar sistemas que identifiquem a personalidade, humor e emoções do usuário. Se um sistema precisa reagir de forma individualizada para cada usuário, personalidade, emoções o primeiro passo seria medir como esse usuário se sente ao utilizar aquele sistema (NUNES, 2012).

Dentro desse contexto surgem os questionários que medem não somente a cognição, mas também as emoções. Mantova (MANTOVA, 2018) comparou onze métodos para avaliar de que forma as emoções são afetadas ao ter contato com uma interface.

Figura 1 - Estudo Comparativo dos métodos

\begin{tabular}{|c|c|c|c|c|c|}
\hline \multirow{2}{*}{\multicolumn{2}{|c|}{$\begin{array}{l}\text { Sigla ou } \\
\text { Nome do Instrumento: }\end{array}$}} & \multicolumn{4}{|c|}{ Formato } \\
\hline & & \multirow{2}{*}{$\frac{\text { Eixo " } x \text { " }}{\text { Não }}$} & \multirow{2}{*}{$\frac{\text { Eixo "y" }}{\text { Não }}$} & \multirow{2}{*}{$\frac{\text { Eixo " } x " e^{\prime \prime} " y "}{\operatorname{Sim}}$} & \multirow{2}{*}{$\frac{\text { Lúdico }}{\text { Não }}$} \\
\hline 1 & Affect Grid & & & & \\
\hline 2 & ATTRAKDIFF & Sim & Não & Não & Não \\
\hline 3 & GAQ & Sim & Não & Não & Não \\
\hline 4 & GEW & Não & Não & Sim & Não \\
\hline 5 & IMI & Sim & Não & Não & Não \\
\hline 6 & Mental Effort & Não & Sim & Não & Não \\
\hline 7 & PANAS & Sim & Não & Não & Não \\
\hline 8 & Presence Questionnaire & Sim & Não & Não & Não \\
\hline 9 & SAM & Não & Não & Não & Sim \\
\hline 10 & SUMI & Sim & Não & Não & Não \\
\hline 11 & WAMMI & Sim & Não & Não & Não \\
\hline
\end{tabular}

Fonte: (MANTOVA, 2018).

Note que apenas dois métodos da Erro! Fonte de referência não encontrada. levam em conta os eixos $\mathrm{X}$ - Prazer e $\mathrm{Y}$ - Desprazer ao mesmo tempo, exatamente o que se pretende avaliar neste trabalho. Porém o Affect Grid (RUSSELL, WEISS e MENDELSOHN, 1989) propõe uma escala objetiva adequada para estudos que exijam julgamentos afetivos do tipo descritivo ou subjetivo, o que não é do interesse desse trabalho.

O GEW (SACHARIN, SCHLEGEL e SCHERER, 2012) é o método escolhido para esta pesquisa por apresentar 40 (quarenta) emoções agrupadas em 20 (vinte) possibilidades de resposta com cinco níveis de percentual em cada uma delas levando em conta eixo "X" e eixo "Y".

\section{MATERIAL E MÉTODOS}


O objetivo geral ou primário é aferir o quanto a usabilidade de ferramentas assistivas afeta a emoção e também a cognição. A ferramenta escolhida foi o SCALA desenvolvido e mantido pela Universidade Federal do Rio Grande do Sul (BITTENCOURT e FUMES, 2016) . A ferramenta SCALA foi escolhida porque é a única online e gratuita disponível no Brasil.

Para mensurar como cada uma das personalidades do ICP reage cognitivamente e emocionalmente ao uso de ferramentas assistivas, afim de entender se esses fatores podem ser considerados pelos desenvolvedores de interfaces assistivas, no momento da criação de software.

A metodologia escolhida para a pesquisa é do tipo qualitativa, de caráter exploratório e fundamentado em raciocínio indutivo. A pesquisa bibliográfica começa a partir de uma estrutura interpretativa/teórica que contextualiza o estudo da pesquisa. A coleta de dados foi feita pelo Google Forms e a modelagem deles, será descrito na seção Resultados (CRESWELL, 2014). Critérios de Inclusão para participação na pesquisa:

1. É preciso saber usar o sistema operacional Windows em um nível médio a. copiar e colar arquivos e pastas;

2. É preciso assinar digitalmente o termo de compromisso, com as instruções para o experimento;

3. Saber ler e escrever;

4. Ter mais de 18 anos;

5. Os participantes não precisam possuir necessidades especiais.

Critérios de Exclusão

1. Ser menor de 18 anos;

2. Não saber ler ou escrever;

3. Ser completamente leigo ao uso de computadores e internet;

A execução, foi construir um formulário no Google Forms, com sessenta e seis questões, dentre elas:

1. Nove perguntas para classificar os participantes: nome, e-mail, concordância com a pesquisa, idade, escolaridade, experiencia com informática e se era ou não da área de Tecnologia da Informação;

2. Vinte e sete perguntas, escala Likert, para determinar a personalidade ICP; 
3. Vinte perguntas, escala Likert, para determinar as emoções GEW;e

4. Dez perguntas, escala Likert, para determinar o uso cognitivo SUS.

O uso das redes sociais, contatos, aplicativos de mensagens, foi o caminho percorrido para se chegar a 96 participantes.

\section{PREPARO DOS DADOS}

Um banco de dados usando triggers e procedures, usa um algoritmo do método ICP cedido gentilmente pelo professor Cristiano (GOMES, 2012), para classificar os participantes dentre uma das oito personalidades que o método mapeia: Abertura a novas experiencias, Extroversão, Mutabilidade, Estabilidade, Foco em Relações, Introversão, Foco no Objetivo e Foco no Objeto.

A seguir processa-se o GEW: é feito uma média das dez emoções de valência positiva (eixo "X") e subtraído das médias das 10 emoções de valência negativa (eixo "Y") onde o resultado máximo pode ser no máximo cinco e no mínimo cinco negativo. O SUS, é calculado, usando-se o algoritmo abaixo:

1. Para as respostas ímpares $(1,3,5)$, subtraia 1 da pontuação que o usuário respondeu;

2. Para as respostas pares ( 2 e 4 ), subtraia a resposta de 5. Ou seja, se o usuário respondeu 2, contabilize 3;

3. Se o usuário respondeu 4, contabilize 1;

4. Agora some todos os valores das dez perguntas, e multiplique por 2.5;

5. Essa é sua pontuação final, que pode ir de 0 a 100.

A média do System Usability Score é sessenta e oito pontos, quanto mais baixo, mais indica que o usuário achou difícil a tarefa, ou seja que a usabilidade da ferramenta é baixa. Depois do processamento, dos sessenta e seis campos, tem se o seguinte resumo: Tipo ICP, Valor do SUS, Valor GEW.

\section{RESULTADOS}

Considerando-se os critérios de inclusão abrangem uma parte significativa da população brasileira de cerca de 220 (duzentos e vinte) milhões de pessoas, assume-se um espaço amostral enumerável de cardinalidade infinita. Dessa maneira, a dimensão n da 
amostra com significância estatística de $5 \%$ foi obtida por meio da fórmula definida por,

$$
n=\frac{z_{\alpha}^{2} \times \hat{p} \times(1-\hat{p})}{e^{2}}
$$

em que $\hat{p}$ representa a proporção que estima-se encontrar na população, $z_{\alpha}$ o quantil da distribuição normal padrão associado ao nível de significância $(\alpha)$ fixado e $e$ a margem de erro máxima admitida. Neste estudo, adotamos $\hat{p}=1 / 2$ a fim de garantir que o tamanho amostral atenderá qualquer que seja a prevalência encontrada, uma vez que a variabilidade será maximizada. Como $\alpha=5 \%$, tem-se que $z_{0}=1,96$. Fixou-se também $e=$ $10 \%$, obtendo $n=96,04$. Portanto, a amostra foi composta por 96 indivíduos.

Com base nas respostas dos 96 indivíduos amostrados, foram encontrados quatro dos oito perfis de personalidade possíveis: Estabilidade, Extroversão, Foco no Objetivo e Mutabilidade. Por meio dos gráficos da Fonte: podemos observar a existência de diferenças entre os grupos de personalidade, no que se refere aos índices GEW e SUS, tanto em termos de variabilidade quanto de tendência central. Para avaliar a significância destas variações, foi realizada uma análise de variância (ANOVA), cuja metodologia consiste em determinar se as médias populacionais de dois ou mais grupos diferem entre si.

Foram ajustados dois modelos; o primeiro considerando o índice GEW como variável resposta e a personalidade como fator que, portanto, é composto por quatro níveis. Analogamente, ajustou-se o modelo considerando o índice SUS como variável resposta. É válido ressaltar que, por ser um método paramétrico, algumas suposições são feitas para o ajuste do modelo: (1) as populações são normalmente distribuídas, (2) possuem variâncias homocedásticas e (3) as observações da amostra são independentes entre si.

Os resultados para o modelo com resposta GEW foram omitidos, uma vez que o pressuposto de normalidade foi violado (valor $p=0,0304$ segundo o teste ShapiroWilk para normalidade, referente ao grupo de personalidade Mutabilidade). 
Figura 2 - Boxplots para os índices GEW e SUS com a personalidade encontrada.
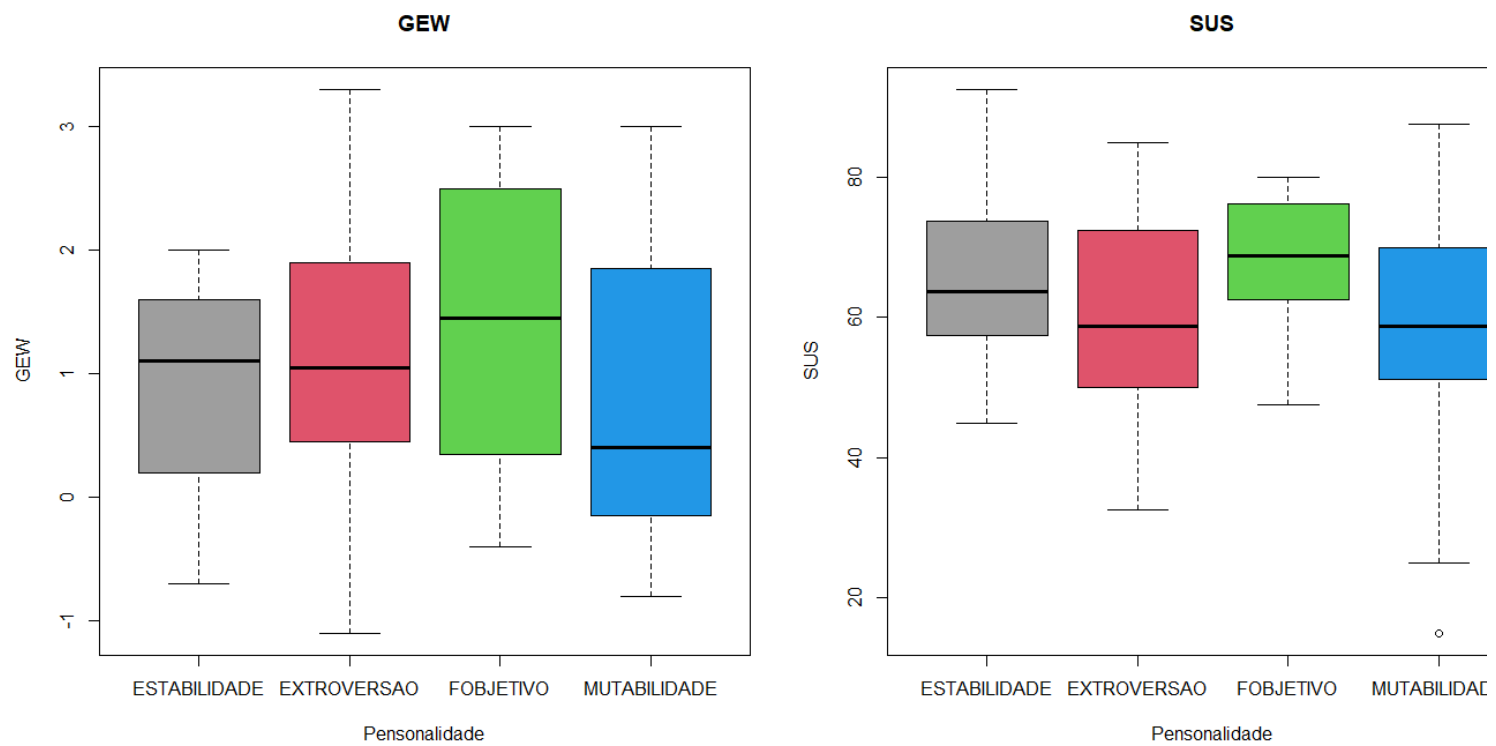

Fonte: Andrade, 2022.

Em relação ao índice SUS, a Fonte: Andrade, 2022.. exibe os resultados obtidos pela ANOVA. Considerando 5\% de nível de significância, tem-se que nenhum dos grupos apresenta diferença significativa, embora o valor $\mathrm{p}$ para o grupo Mutabilidade (valor $p=$ 0,0675) tenha ficado relativamente próximo do valor de referência.

Tabela 1 - Resultados da ANOVA para o modelo com resposta SUS.

\begin{tabular}{|l|r|r|r|r|}
\hline & Estimativa & \multicolumn{1}{|l|}{ Erro padrão } & \multicolumn{1}{l|}{ T } & \multicolumn{1}{l|}{ Valor $\mathrm{p}$} \\
\hline Intercepto & 65,75 & 3,14 & 20,89 & $<2 \mathrm{e}-16$ \\
\hline ICP extroversão & $-7,00$ & 4,01 & $-1,74$ & 0,0844 \\
\hline ICP fobjetivo & 1,59 & 4,72 & 0,33 & 0,7364 \\
\hline ICP mutabilidade & $-7,62$ & 4,12 & $-1,85$ & 0,0675 \\
\hline
\end{tabular}

Fonte: Andrade, 2022.

Para verificar os pressupostos da análise de variância, foram realizados os testes descritos Fonte: Andrade, 2022 Considerando 5\% de significância, nenhuma das hipóteses dos três testes realizados foi rejeitada, sugerindo, portanto, que não há falhas de especificação do modelo com variável resposta SUS. 
Tabela 2 -Resultados dos testes para verificação das pressuposições do modelo com reposta SUS.

\begin{tabular}{|c|c|}
\hline Teste Shapiro-Wilk para normalidade & Valor $\mathbf{p}$ \\
\hline \multicolumn{2}{|c|}{$\boldsymbol{H}_{\mathbf{0}}:$ os dados possuem distribuição normal } \\
\hline Estabilidade & 0,6927 \\
\hline Extroversão & 0,3827 \\
\hline Foco no Objetivo & 0,1854 \\
\hline Mutabilidade & 0,4240 \\
\hline Teste de Bartlett para homocedasticidade ${ }^{1}$ & Valor p \\
\hline $\boldsymbol{H}_{\mathbf{0}}$ : variâncias homocedásticas & 0,1494 \\
\hline Teste de Durbin-Watson para independência & Valor p \\
\hline $\boldsymbol{H}_{\mathbf{0}}:$ as observações são independentes & 0,7660 \\
\hline
\end{tabular}

Fonte: Andrade, 2022.

Alternativamente à ANOVA, foi realizado o teste não paramétrico de KruskalWallis, cuja hipótese nula é dada por

$H_{0}$ : não existe diferença entre a distribuição dos grupos.

Novamente, a metodologia foi utilizada considerando os dois índices separadamente. Tanto para o índice GEW, quanto para o índice SUS, os valores de p encontrados $(0,2189$ e 0,0874, respectivamente) indicam que os índices possuem a mesma distribuição de probabilidade dentro dos 4 grupos de personalidade, considerando 5\% de significância.

Com o intuito de verificar a existência de correlação entre os índices GEW e SUS na amostra, calculou-se o coeficiente de correlação de postos de Spearman, avaliando, portanto, a intensidade da relação entre as duas variáveis. Tal quantidade varia no intervalo $[-1,1]$, indicando forte associação à medida que o coeficiente se aproxima de $1 \mathrm{em}$ módulo.

\footnotetext{
${ }^{1}$ A homocedasticidade descreve uma situação em que o termo de erro (ou seja, o "ruído" ou perturbação aleatória na relação entre as variáveis independentes e a variável dependente) é o mesmo em todos os valores das variáveis independentes.
} 
O sinal indica se a correlação é positiva ou negativa, isto é, proporcional ou inversamente proporcional. Como resultado, obteve-se $\rho=0,4264$ com valor $p=1,479 e-$ 05, o que revela uma correlação moderada positiva e significativa, uma vez que se rejeita a hipótese nula do teste (não existência de associação monotônica entre as variáveis). O gráfico de dispersão, vide Fonte: Andrade, 2022 corrobora com o supramencionado.

Figura 3 - Gráfico de dispersão entre as variáveis GEW e SUS, classificados por personalidade.

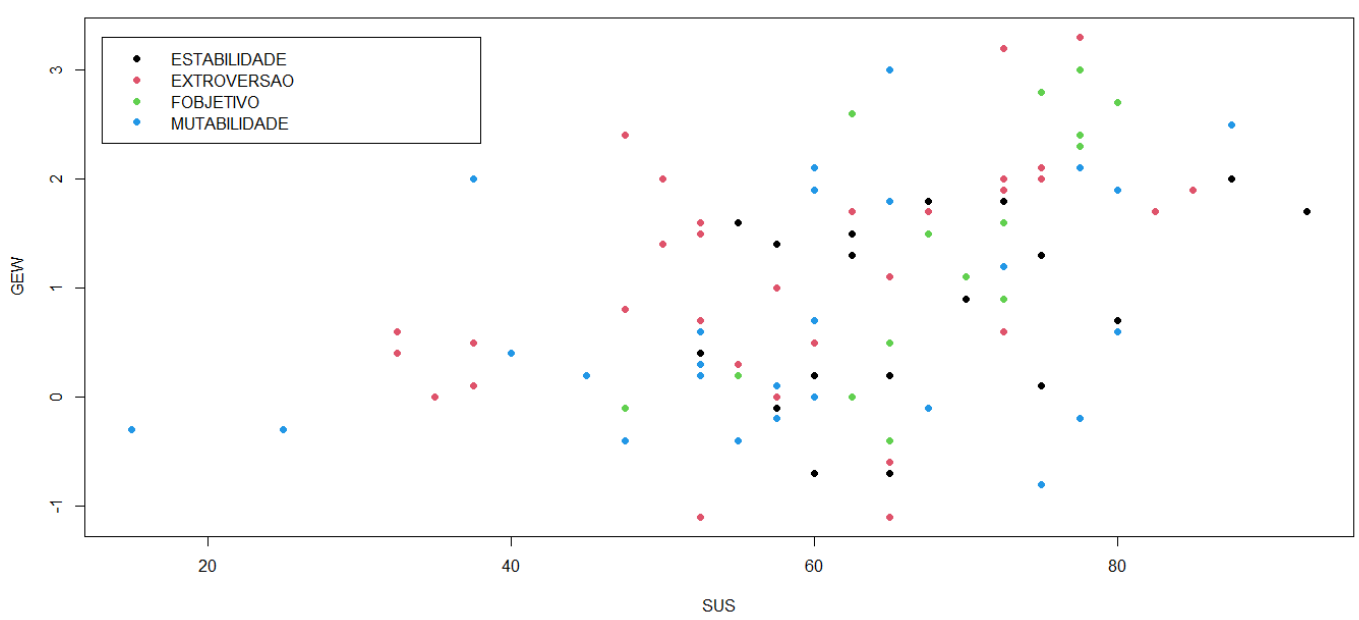

Fonte: Andrade, 2022

\section{CONCLUSÕES}

Neste trabalho, investigou-se a relação entre as diferentes personalidades e suas reações emocionais e cognitivas ao utilizar uma ferramenta assistida. Buscou-se, principalmente, verificar se usuários com o mesmo tipo de personalidade apresentam a mesma percepção emocional ou cognitiva sobre a usabilidade de interfaces assistivas, bem como, se as percepções emocional e cognitiva, no uso de uma ferramenta assistiva, tem correlação entre si.

Não foram encontradas evidências estatísticas para acreditar na relação entre às personalidades e às percepções emocionais e cognitivas, conforme o obtido por meio da ANOVA e do teste de Kruskal-Wallis. Contudo, os valores de preferentes às análises com os índices de percepção cognitiva nos levam a pensar que tais resultados poderiam ser diferentes neste âmbito, caso o tamanho amostral fosse maior ou a amostra fosse mais heterogênea entre si, logo: 
H1: Nulo, sob o prisma de duas metodologias ANOVA e Kruskal-Wallis;

O valores de $\mathrm{p}$ referentes às análises com os índices de percepção cognitiva nos levam a pensar que tais resultados poderiam ser diferentes neste âmbito, caso o tamanho amostral fosse maior ou a amostra fosse mais heterogênea entre si ou ainda se grau de significância fosse ajustado, e isso depende de uma série de fatores inclusive da visão do pesquisador (MYERS, WELL e LORCH JR, 2013), se ele fosse ajustado para $10 \%$ ao invés de 5\%, tanto na metodologia ANOVA quanto no teste de Teste de Kruskal-Wallis, teríamos significância estatística para a $\mathrm{H} 2, \operatorname{logo}$ :

H2: Pode ser aceito se a significância for aumentada sob o prisma de duas metodologias ANOVA e Kruskal-Wallis ou ainda deve ser melhor investigado alterando/aumentando a população.

Paralelamente, a análise de correlação indicou a existência de uma associação positiva monotônica entre as variáveis GEW e SUS na população, embora seja de intensidade moderada. Pelo gráfico de dispersão, pode-se verificar que os valores do índice GEW crescem à medida que os valores SUS também aumentam, porém com uma variabilidade relativamente alta, então:

H3: Positiva, moderada, segundo o Coeficiente de Correlação de postos de Spearman.

\section{TRABALHOS FUTUROS}

Essa pesquisa poderia ter abrangido um número maior e mais diversificado de pessoas se não fosse a COVID-19. Assim, após o mundo voltar ao seu "normal" a facilidade de conseguir com que cem, ou mais, novos participantes participem e usar os modelos já construídos aqui, é uma proposta de trabalho futuro.

Uma parceria com empresas que produzem software assistivo para permitirem que sua interface seja testada nos moldes desse trabalho é uma continuidade que traria contribuição à ciência e à indústria de software.

Em suma com uma única amostra de noventa e seis pessoas usando métodos consistentes ficou claro que essa é uma linha de pesquisa a ser explorada quer seja trocando/aumentando o público, trocando a ferramenta assistiva que foi testada ou ainda testando outros métodos emocionais e cognitivos que não sejam o SUS ou GEW. 


\section{REFERENCIAS}

ABRAS, C. et al. User-centered design. Bainbridge, W. Encyclopedia of HumanComputer Interaction. Thousand Oaks: Sage Publications, v. 37, p. 445-456, 2004.

ANDRADE, J. M. D. Evidências de validade do inventário dos cinco grandes fatores de personalidade para o Brasil, 2008.

ANDRIOLA, W. B. Psicometria Moderna: características e tendências. Estudos em Avaliação Educacional, v. 20, p. 319-340, 2009.

ASSILA, A.; EZZEDINE, H.; OTHERS. Standardized usability questionnaires: Features and quality focus. Electronic Journal of Computer Science and Information Technology, v. 6, 2016.

BERCHT, M. Computação Afetiva: vínculos com a psicologia e aplicações na educação. Produções do III PSICOINFO e II JORNADA do NPPI, p. 106, 2006.

BITTENCOURT, I.; FUMES, N. A tecnologia assistiva SCALA na promoção de narrativas de sujeitos com Transtorno do Espectro Autista sobre as suas experiências escolares e o autismo. Brazilian Symposium on Computers in Education (Simpósio Brasileiro de Informática na Educação-SBIE). [S.1.]: [s.n.]. 2016. p. 767.

BRIGGS, K. C. Myers-Briggs type indicator. [S.1.]: Consulting Psychologists Press Palo Alto, CA, 1976.

CESA, C. C.; MOTA, H. B. Comunicação aumentativa e alternativa: panorama dos periódicos brasileiros. Revista CEFAC, v. 17, p. 264-269, 2015.

CRER. Centro Estadual de Reabilitação e Readaptação Dr Henrique Santillo, Página inicial. Disponível em: < https://www.agirsaude.org.br/> . Acesso em: 10 de dez. de 2021.

COELHO, F. G. D. M. et al. Desempenho cognitivo em diferentes níveis de escolaridade de adultos e idosos ativos. Revista Brasileira de Geriatria e Gerontologia, v. 15, p. $7-$ $15,2012$.

CRESWELL, J. W. Investigação Qualitativa e Projeto de Pesquisa-: Escolhendo entre Cinco Abordagens. [S.1.]: Penso Editora, 2014. 
CUNHA, L. M. A. D.; OTHERS. Modelos Rasch e Escalas de Likert e Thurstone na medição de atitudes. [S.1.]. 2007.

DAMASIO, A. Damasio, Antonio R., Descartes' Error: Emotion, Reason, and the Human Brain. Relations industrielles, 1995.

DESOUSA, D. A. et al. Revisão sistemática de instrumentos para avaliação de ansiedade na população brasileira. Avaliação Psicológica, v. 12, p. 397-410, 2013.

FIGUEIREDO FILHO, D. B.; SILVA JÚNIOR, J. A. Desvendando os Mistérios do Coeficiente de Correlação de Pearson (r). Revista Política Hoje, v. 18, p. 115-146, 2009.

FLORES-MENDOZA, C. Estudo brasileiro do NEO-FFI-R (versão curta). NEO PI-R Inventário de personalidade NEO revisado e inventário de cinco fatores NEO revisado NEO-FFI-R (versao curta), v. 1, p. 93-104, 2007.

FURNHAM, A.; CHAMORRO-PREMUZIC, T.; MCDOUGALL, F. Personality, cognitive ability, and beliefs about intelligence as predictors of academic performance. Learning and individual Differences, v. 14, p. 47-64, 2003.

GILlHAM, N. W. Sir Francis Galton and the birth of eugenics. Annual review of genetics, v. 35, p. 83-101, 2001.

GOMES, C. M. A. A estrutura fatorial do inventário de características da personalidade. Estudos de Psicologia (Campinas), v. 29, p. 209-220, 2012.

GOMES, C. M. A. A estrutura fatorial do inventário de características da personalidade. Estudos de Psicologia (Campinas), v. 29, p. 209-220, 2012.

GOMES, C. M. A.; GJIKURIA, E. Comparing the ESEM and CFA approaches to analyze the Big Five factors. Avaliação Psicológica, v. 16, p. 261-267, 2017.

HARPER, B. D.; NORMAN, K. L. Improving user satisfaction: The questionnaire for user interaction satisfaction version 5.5. Proceedings of the 1st Annual Mid-Atlantic Human Factors Conference. [S.1.]: [s.n.]. 1993. p. 224-228.

INSTITUTO BRASILEIRO DE GEOGRAFIA E ESTATÍSTICA. Censo demográfico 
2010. Rio de Janeiro, RJ, 2010. Disponível em: <http://www.ibge.gov.br/home/presidencia/noticias/imprensa/ppts/00000008473104122012315727483985.pdf>. Acesso em: 10 de dez. de 2021.

KIRAKOWSKI, J.; CORBETT, M. SUMI: The software usability measurement inventory. British journal of educational technology, v. 24, p. 210-212, 1993.

LAMIELL, J. T. William Stern: More than" the IQ guy". Annual Convention of the American Psychological Association, 99th, Aug, 1991, San Francisco, CA, US; This chapter is a revision of a paper presented at the aforementioned conference. [S.1.]: [s.n.]. 1996.

LEOTTI, V. B.; COSTER, R.; RIBOLDI, J. Normalidade de variáveis: métodos de verificação e comparação de alguns testes não-paramétricos por simulação. Revista HCPA. Porto Alegre. Vol. 32, no. 2 (2012), p. 227-234, 2012.

LEWIS, J. R. Measuring perceived usability: The CSUQ, SUS, and UMUX. International Journal of Human-Computer Interaction, v. 34, p. 1148-1156, 2018.

LIKERT, R. A technique for the measurement of attitudes (Archives of Psychology, No: 140). New York City: Columbia University, v. 7, 1932.

LIMA, M. M. B. M. P. D. NEO-PI-R-Contextos Teóricos e Psicométricos:" Ocean" ou" Iceberg". [S.1.]. 1997.

MANTOVA, A. R. D. Design e emoção: aplicação do Círculo das Emoções de Genebra para a avaliação emocional durante a experiência do usuário, 2018.

MARSH, H. W. et al. Exploratory structural equation modeling: An integration of the best features of exploratory and confirmatory factor analysis. Annual review of clinical psychology, v. 10, p. 85-110, 2014.

MATISE, M. The enneagram: An enhancement to family therapy. Contemporary Family Therapy, v. 41, p. 68-78, 2019.

MORAIS, F. et al. Computação Afetiva aplicada à Educação: uma revisão sistemática das pesquisas publicadas no Brasil. Brazilian symposium on computers in education (simpósio brasileiro de informática na educação-sbie). [S.1.]: [s.n.]. 2017. p. 163. 
MYERS, J. L.; WELL, A. D.; LORCH JR, R. F. Research design and statistical analysis. [S.1.]: Routledge, 2013.

NARANJO, C. El Eneagrama de la Sociedad. Males del mundo, males del alma. [S.1.]: JC Sáez Editor, 1995.

NIELSEN, J. Evaluating hypertext usability. In: Designing hypermedia for learning. [S.1.]: Springer, 1990. p. 147-168.

NU, Nações Unidas, A ONU e as pessoas com deficiência. Disponível em: <https://nacoesunidas.org/acao/pessoas-com-deficiencia/>. Acesso em: 10 de dez. de 2021.

NUNES, C. H. S. S.; HUTZ, C. S.; NUNES, M. F. O. Bateria Fatorial de Personalidade (BFP): manual técnico. São Paulo: Casa do Psicólogo, v. 126, 2010.

NUNES, M. A. S. N. Computação Afetiva personalizando interfaces, interações e recomendações de produtos, serviços e pessoas em Ambientes computacionais. DCOMP e PROCC: Pesquisas e Editora UFS: São Cristóvão, p. 115-151, 2012.

PALMER, H. The enneagram. [S.1.]: Harper San Francisco, 1991.

PARREIRA, S. L. S. et al. Equipe Interdisciplinar para Utilização de Tecnologias de Comunicação Alternativa e Aumentativa. Fronteiras: Journal of Social, Technological and Environmental Science, v. 4, p. 334-342, 2015.

PEREIRA, R. IHC na Especialização em Engenharia de Software: discussões sobre uma experiência prática. WEIHC. [S.1.]: [s.n.]. 2013. p. 1-6.

PICARD, R. W. Affective computing. [S.1.]: MIT press, 2000.

REGAZZI, A. J. Teste para verificar a identidade de modelos de regressão e a igualdade de alguns parâmetros num modelo polinomial ortogonal. Ceres, v. 40, 2015.

RUSSELL, J. A.; WEISS, A.; MENDELSOHN, G. A. Affect grid: a single-item scale of pleasure and arousal. Journal of personality and social psychology, v. 57, p. 493, 1989.

SACHARIN, V.; SCHLEGEL, K.; SCHERER, K. R. Geneva emotion wheel rating study, 2012. 
SIEGLER, R. S. The other Alfred Binet. Developmental psychology, v. 28, p. 179, 1992.

SIRKIN, R. M. Two-sample t tests. Statistics for the social sciences, p. 271-316, 2005.

SMITH, C.; MAYES, T. Telematics applications for education and training: usability guide. Comission of the European Communities, DGXIII Project, 1996.

SZWARCWALD, C. L.; DAMACENA, G. N. Amostras complexas em inquéritos populacionais: planejamento e implicações na análise estatística dos dados. Revista Brasileira de Epidemiologia, v. 11, p. 38-45, 2008.

TRAPPL, R.; PETTA, P. Facets of emotions in humans and artifacts. In: Data Fusion and Perception. [S.1.]: Springer, 2001. p. 171-181.

TRÓCCOLI, B. T. et al. Inventário Reduzido dos Cinco Grandes Fatores de Personalidade [Resumo]. Anais do II Congresso Norte Nordeste de Psicologia. Salvador, BA: Editora da Universidade Federal da Bahia. [S.1.]: [s.n.]. 2001.

VIEIRA, S. Bioestatística: Tópicos avançados. ed. Campus, Rio de Janeiro, 2003.

WILSON, R. A.; SHAW, B. The miraculous birth of language. Tijdschrift Voor Filosofie, v. 12, 1950. 